\section{Frühe Tumorentstehung}

Chronische entzündliche Darmerkrankungen stellen einen wichtigen Risikofaktor für die Entstehung von Darmkrebs dar. Jetzt ist einer Arbeitsgruppe aus Erlangen, der Nachweis gelungen, dass das Molekül VEGF („vascular endothelial growth factor") das Tumorwachstum begünstigt. Die Wissenschaftler untersuchten an einem Modell des entzündungsbedingten Darmkrebses bei Mäusen die Rolle von VEGF. Dabei entdeckten sie, dass neben den Gefäß- auch die Tumorzellen Rezeptoren für VEGF besitzen. In weiteren Untersuchungen zeigte die Arbeitsgruppe, dass eine dauerhafte Entzündung die Bindung von VEGF an

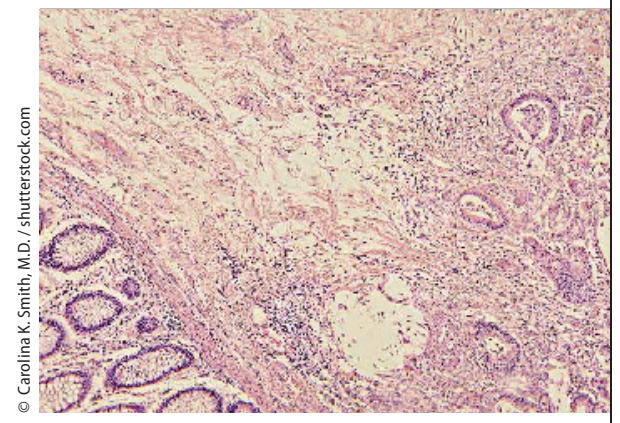

zuvor gesunden Darmzellen ermöglicht. Dabei führte das entzündungsfördernde IL-6 dazu, dass Darmzellen den Rezeptor für VEGF tragen. Bindet nun VEGF an Darmzellen, kann es den Wachstumsfaktor STAT3 aktivieren und dadurch die Tumorentstehung fördern.

Bislang hatte man angenommen, dass VEGF nur an der Gefäßneubildung in bereits bestehenden Tumoren beteiligt ist. Die neuen Daten legen nahe, dass eine Aktivierung von VEGF schon bei sehr frühen Stadien der Tumorentstehung von Bedeutung ist. Weitere Studien zeigen, dass VEGF auch direkt an der Aufrechterhaltung der chronischen Entzündungsreaktion beteiligt ist. Somit könnte eine Hemmung von VEGF zu einer Verringerung der Darmentzündung führen.

Literatur: Waldner MJ, Wirtz S, Jefremow A et al (2010) VEGF receptor signaling links inflammation and tumorigenesis in colitis-associated cancer. J Exp Med, doi: 10.1084/ jem.20100438

Quelle: Universitätsklinikum Erlangen, www.uk-erlangen.de telt. Aus diesen Daten lassen sich die Prävalenzen der Krankheit und der Exposition ermitteln. Querschnittsstudien können damit als Momentaufnahme einer populationsbasierten Kohortenstudie aufgefasst werden. Aufgrund der gleichzeitigen Erfassung von Expositionen und Erkrankungen sind sie besonders für Zwecke der beschreibenden Epidemiologie nützlich [3].

\section{Kohortenstudien}

Epidemiologische Studien zur Ätiologie chronischer Erkrankungen müssen auf dem Einzelpersonenniveau angesiedelt sein, wobei individuelle Erkrankungsund Expositionsdaten erhoben werden, um einen kausalen Zusammenhang zwischen dem Eintreten der Erkrankung und einer zeitlich oft weit davor liegenden Exposition herzustellen. Wird das Vorliegen oder Nichtvorliegen einer interessierenden Exposition zuerst erhoben und ausgehend davon das Eintreten oder Nichteintreten der Krankheit prospektiv beobachtet, spricht man von einer Kohortenstudie. Es handelt sich demzufolge um längsschnittmäßige Beobachtungsstudien.

Bei einer rein prospektiven Kohortenstudie wird ausgehend von einer aktuellen Erhebung der interessierenden Exposition die Kohorte (Studienpopulation) prospektiv in die Zukunft im Hinblick auf das Eintreten der relevanten Gesundheitsereignisse verfolgt [13]. Ein Beispiel für einen solchen Studientyp ist die so genannte EPIC-Studie („European Prospective Investigation into Cancer and Nutrition"). Hier wurden zwischen 1992 und 1998 in 10 europäischen Ländern bei 366.521 Frauen und 153.457 Männern die Ernährungsgewohnheiten systematisch erfasst. In einer mehrjährigen Nachbeobachtungsphase („follow up“) wurde das Auftreten von Krebserkrankungen ermittelt. Es zeigte sich, dass ein höherer Konsum von Ballaststoffen mit einem erniedrigten Risiko für das Kolorektalkarzinom einhergeht. Es wurde ein signifikantes relatives Risiko (RR) von 0,75 [95\%-Konfidenzintervall (95\%-CI) 0,590,95] für die höchste vs. niedrigste Kate- gorie der Ballaststoffaufnahme ermittelt $[2,5]$.

Das relative Risiko ist die Relation der Erkrankungswahrscheinlichkeiten von Exponierten und Nichtexponierten. Ein RR $>1$ gibt an, dass die Gruppe der Exponierten ein höheres Erkrankungsrisiko hat als die der Nichtexponierten. Ein relatives Risiko von beispielsweise 1,2 ist mit einer 20\%igen Risikoerhöhung für die Exponierten gleichzusetzen, ein RR von o,8 mit einer entsprechenden Risikominderung. Das Konfidenzintervall für das relative Risiko wird ermittelt, um die Präzision der Schätzung angeben zu können und eine Aussage über die statistische Signifikanz des Ergebnisses zu ermöglichen [8].

\section{Fall-Kontroll-Studien}

Während Kohortenstudien bei zunächst krankheitsfreien Personen ausgehend von einer spezifischen Exposition das Auftreten (mehrerer) Krankheiten oder Gesundheitsereignisse untersuchen, wird umgekehrt in Fall-Kontroll-Studien bei Erkrankten bzw. Nichterkrankten die vorausgegangene Expositionshäufigkeit festgestellt. Fall-Kontroll-Studien gehen somit von einer definierten Krankheit aus und streben an, wichtige Informationen über ätiologisch relevante Expositionen retrospektiv zu erheben.

Bei diesem Studientyp werden 2 Kollektive miteinander verglichen. Bei den Fällen ist die Krebserkrankung aufgetreten, bei den so genannten Kontrollen nicht. Letztere werden häufig im Verhältnis zu Ersteren so ausgewählt, dass sie diesen in bestimmten Grunddaten, z. B. Alter, Geschlecht, entsprechen (Matching), wodurch die statistische Effizienz bei der Kontrolle von Störfaktoren (Confounder) in der Auswertung erhöht werden soll [3]. Kontrollpersonen können Menschen aus der Bevölkerung sein oder aber Krankenhauspatienten, die wegen einer anderen Krankheit eingewiesen wurden als die Fälle. Nachteilig bei Krankenhauskontrollen ist allerdings, dass es sich um eine Stichprobe aus einer unscharf definierten Bezugspopulation handelt. Darüber hinaus unterscheiden sie sich von der Allgemeinbevölkerung. So ist z. B. bekannt, 